\title{
Rechtsfragen im Zusammenhang mit Milchbanking
}

Brugger Schmidt, Caroline ; Gächter, Thomas

Posted at the Zurich Open Repository and Archive, University of Zurich ZORA URL: https://doi.org/10.5167/uzh-17778

Journal Article

Originally published at:

Brugger Schmidt, Caroline; Gächter, Thomas (2008). Rechtsfragen im Zusammenhang mit Milchbanking. Revue suisse de droit de la santé = Schweizerische Zeitschrift für Gesundheitsrecht, 11:125-136. 
Weblaw / Institut de droit de la santé de l'Université de Neuchâtel (Editeurs/Hrsg.)

\section{Revue suisse de droit de la santé Schweizerische Zeitschrift für Gesundheitsrecht}

RSDS/SZG 11/2008

Les articles de la présente revue ont déjà été publiés dans la revue en ligne Jusletter (www.jusletter. ch). Sauf indication contraire, les articles correspondent en tout point à la publication en ligne. II est donc conseillé de suivre la proposition de citation de leur première publication.

Die Beiträge der vorliegenden Zeitschrift sind bereits online als Jusletter-Beiträge (www.jusletter.ch) erschienen. Die abgedruckten Beiträge sind - wo nicht anders vermerkt - inhaltlich und bezüglich der Randziffern mit den Online-Beiträgen identisch. Es empfiehlt sich deshalb, die Beiträge entsprechend dem Zitiervorschlag der Erstpublikation nach Randziffern zu zitieren. 


\section{Rechtsfragen im Zusammenhang mit Milchbanking}

In Frauenmilchbanken wird die überschüssige Milch von Frauen pasteurisiert und als Nahrung für Frühgeborene und Säuglinge mit zu geringem Geburtsgewicht gelagert. Es rechtfertigt sich, den Milchbanken mehr juristische Beachtung zu schenken, da sie einerseits wichtige Leistungen im Interesse der Frühgeborenen erbringen und sich anderseits - im Hinblick auf den Schutz der Frühgeborenen und einen allfälligen Austausch von Frauenmilch zwischen den Institutionen gewisse Sicherheits- und Schutzstandards aufdrängen.

Les banques spécialisées dans la conservation du lait maternel pasteurisent et stockent le lait excédentaire pour nourrir les prématurés et les nouveaux-nés à poids trop faible. D'un point de vue juridique, il se justifie d'accorder plus d'attention à ces banques du lait qui, d'une part, fournissent des prestations importantes aux prématurés et qui, d'autre part, requièrent certains standards de sécurité et de protection en regard de la protection des prématurés et des éventuels échanges de lait maternel inter-institutions. (cli)

Zitiervorschlag: Caroline Brugger Schmidt / Thomas Gächter, Rechtsfragen im Zusammenhang mit Milchbanking, in: Jusletter 13. August 2007 
Inhaltsübersicht

1. Bedeutung der Frauenmilch und der Frauenmilchbanken

1.1. Historische Ursprünge: Ammen

1.2. Bedeutung der Muttermilch respektive Spenderinnenmilch

1.3. Funktionsweise von Frauenmilchbanken

1.4. RechtlicheFragen

2. Gibtes ein Grundrecht aufoptimale Ernährung?

2.1. Völkerrechtliche Betrachtung

2.2. Grundlagen in der schweizerischen Bundesverfassung

2.3. Zwischenfazit

3. Sozialversicherungsrechtliche Regelungen

3.1. Kostenübernahme für den Konsum von Frauenmilch

3.2. Mietkosten für Abpumpgeräte

4. DiejuristischeQualifikationdergespendetenMilchunddierechtlichenGrundlagenfür den Betrieb von Milchbanken

4.1. Sachenrechtliche Qualifikation der Frauenmilch

4.2. Frauenmilch als Arznei-oder als Lebensmittel?

5. Abschliessende Gedanken

Literaturverzeichnis

\section{Bedeutung der Frauenmilch und der Frauenmilchbanken}

\subsection{Historische Ursprünge: Ammen}

[Rz 1] Die Ursprünge der heutigen Milchbanken' reichen in die Zeit zurück, als Kinder, die nicht von ihren Müttern gestillt wurden, durch Freundinnen, Bekannte oder andere Frauen ernährt wurden. Diese sogenannten Ammen stillten anstelle der Mutter das Neugeborene mit ihrer eigenen Milch. Hinweise auf die Existenz von Ammen findet man bereits im Codex Hammurabi. ${ }^{2}$ Darin werden die Attribute einer guten Amme beschrieben. Man glaubte, die physischen, mentalen und emotionalen Charakteristika eines Kindes würden über die Muttermilch übertragen, was eine sorgfältige Auswahl der Amme bedingte. ${ }^{4}$

$\left[\begin{array}{ll}R z & 2\end{array}\right]$ Soziologisch bedeutsam war die Veränderung der Gesellschaftsstruktur der europäischen Oberschicht im 19 und 20. Jahrhundert: Die Mutter war nicht mehr «Dauerpflegeperson» ihrer Kleinstkinder. An ihre Stelle trat die Amme. Für das heranwachsende Kind ergab sich eine besondere Vertrauensbeziehung zur Amme. Die Verdienstmöglichkeiten einer Amme waren über Jahrhunderte hinweg überdurchschnittlich gut. ${ }^{5}$ In Bern wurden die letzten Berufsammen um 1950 in den Ruhestand geschickt. ${ }^{6}$

[Rz 3] Zu Beginn des 20. Jahrhunderts wurden beinahe sämtliche Kleinkinder mit menschlicher Milch versorgt; entweder durch die Mutter, die Amme oder - etwas später - durch gespendete Milch aus Milchbanken. Diese wurden vor allem in der ehemaligen

\footnotetext{
Der Betrieb von Milchbanken wird auch «Milchbanking» genannt.

2250 vor Christus.

Milch, die von der eigenen Mutter stammt.

4 www.hmbana.org/index.php?mode=history (besucht am 11.7.07).

www.hmbana.org/index.php?mode=history (besucht am 11.7.07).

6 http://de.wikipedia.org/wiki/Amme (besucht am 11.7.07).
}

DDR und in Frankreich eingerichtet. Das letzte Jahrhundert führte jedoch auch im Bereich der Ernährung zu Umwälzungen. Durch die Entwicklung künstlicher Säuglingsmilch und deren kommerzieller Vermarktung sowie durch die Angst, Krankheiten könnten über die Muttermilch übertragen werden, wurde die natürliche Art der Säuglingsernährung zunehmend verdrängt.

[Rz 4] Heute wird wiederum die ausschliessliche Ernährung durch Muttermilch für mindestens sechs Monate propagiert. Es wird gar empfohlen, das Kind für weitere zwei Jahre ergänzend zur altersentsprechenden Nahrung zu stillen. ' Stillen ist nach der vorherrschenden Meinung die ideale Grundlage für ein gesundes Wachstum und die Entwicklung des Kindes. ${ }^{8}$ Stillen hat zudem wichtige Implikationen für die Gesundheit der Mutter. ${ }^{9}$ Ausschliessliches Stillen reduziert die Kindersterblichkeit auf Grund normaler Kinderkrankheiten ${ }^{10}$ und beschleunigt den Genesungsprozess im Fall einer Erkrankung." Stillen hat insgesamt sowohl wirtschaftliche, soziologische als auch gesundheitsspezifische Auswirkungen, die meist positiv zu bewerten sind.

\subsection{Bedeutung der Muttermilch respektive Spende- rinnenmilch}

[Rz 5] Gerade beiKindern, die aufGrund ihres Allgemeinzustandes eine optimale und natürliche Ernährung besonders nötig hätten, besteht wegen schwieriger Gesamtumstände häufig die Gefahr, dass sie mit künstlicher Milch oder suboptimaler Nahrungsergänzung ernährt werden. Zu denken ist etwa an untergewichtige Termingeborene ${ }^{12}$ oder an Frühgeborene. ${ }^{13,1}$, Ein direktes Stillen kann aber auch auf Grund pathologischer Eigenschaften des Babys unmöglich sein. ${ }^{15}$ Gründe für Flaschenernährung finden sich viele. ${ }^{16}$ Muttermilch ist aber insbesondere in Bezug auf ihre Verträglichkeit, die enthaltenen Wachstumsfaktoren und gastrointestinalen ${ }^{17}$ Verdauungsenzyme sowie der antiinfektiösen Komponenten eine optimale Grundlage für den Aufbau Frühgeborener, kranker Neugeborener, von Säuglingen nach Darmoperationen oder von Kindern mit

\footnotetext{
Ausführlich dazu s. WHO, Breastfeeding, S. 3 ff.

8 Vgl. z.B. Singhal/Cole/Luca, S. 413; Work Group on Breastfeeding, S. 1035.

9 Z.B. beschleunigte Rückbildung nach der Schwangerschaft, verringertes Risiko, später an Brustkrebs zu erkranken; Work Group on BREASTFEEdING, S. 1035; WHO, Breastfeeding, S. 3.

10 Z.B. Durchfall, Lungenentzündung.

11 Kramer M. et AL, S. 414; Both, S. 4.

12 Bei Jungen sollte das Geburtsgewicht ungefähr zwischen 3300 und 3600 Gramm liegen, bei Mädchen zwischen 3160 und 3450 Gramm, BFS, S. 12.

132004 sind 9\% (5674) aller Lebendgeborenen vor der beginnenden 38 Schwangerschaftswoche zur Welt gekommen. Bei diesen Kindern spricht man von Frühgeborenen, BFS, S. 9 ff.

14 Vgl. auch SPRINGER, S. 7 ff.

15 Z.B. auf Grund einer Lippen-Kieferspalte.

16 Vgl. auch WIGHT, S. 249; TULLY, S. 75; WHO/UNICEF, S. 10

17 Den Magen-Darm-Trakt betreffend.
} 
anderen Gedeihstörungen. ${ }^{18}$ Die Ernährung in den ersten drei Lebensjahren ist massgebend für die lebenslange Gesundheit sowie das allgemeine Wohlbefinden. ${ }^{19}$

$\left[\begin{array}{ll}R z & 6\end{array}\right]$ In diesem Sinn spielen Frauenmilchbanken eine entscheidende Rolle im Erreichen international geforderter und geförderter Ziele zur Gesundheitsförderung von Mutter und Kind. Es erstaunt deshalb nicht, dass einerseits die meisten Kinderkliniken zentraleuropäischer Länder, der USA ${ }^{20}$ sowie Südamerikas Bemühungen unterstützen, die frühgeborenen oder kranken Kinder durch abgepumpte Frauenmilch ${ }^{21} \mathrm{zu}$ ernähren. ${ }^{22}$

\subsection{Funktionsweise von Frauenmilchbanken}

$[R z$ 7] Frauen, die genügend Milch für ihr eigenes Kind haben und einen Überschuss an Muttermilch produzieren, können diese für andere Kinder zur Verfügung stellen. Die Spenderin wird vorgängig über den Prozess der Milchspende orientiert und zu ihrer eigenen Gesundheit befragt. Nach der Aufklärung willigt sie in die Spende und das serologische Testverfahren ein. Wird ihr Blut negativ auf bestimmte Krankheitserreger getestet und entspricht ihr allgemeiner Gesundheitszustand den Vorgaben, kann die Frau als Spenderin aufgenommen werden. Die Daten der Spenderin werden mittels einer Identifikationsnummer verschlüsselt und registriert. Die gespendete Milch wird ebenfalls dokumentiert, mikrobiologisch kontrolliert und anschliessend pasteurisiert, gelagert und bei Bedarf für das entsprechende Empfängerkind aufbereitet. ${ }^{23}$

[Rz 8] Durch den Pasteurisierungsprozess wird das Risiko einer Krankheitsübertragung verringert. ${ }^{24}$ In der Schweiz wird nur pasteurisierte Frauenmilch verwendet. Die Spende erfolgt unentgeltlich. Die aufklärungstechnischen und administrativen Belange rund um die Spende sowie sämtliche Arbeiten, die im Zusammenhang mit der Frauenmilch anfallen, ${ }^{25}$ werden in den Milchbanken ${ }^{26}$ durchgeführt. In der Schweiz werden in

18 Ball/Wright, S. 876; Wight, S. 249; LuCAs/Morley/Cole/Gore, S. 72 . Ausführlich zur Indikation von Spenderinnenmilch BотH, S. 5.

19 WHO, Breastfeeding, S. 3 ff.

20 Zur Geschichte und zum Umgang in der Praxis mit Milchbanken in den USA siehe www.lalecheleague.org/Illeaderweb/LV/LVAprMay00p19. html (besucht am 11.7.07).

${ }^{21}$ Milch, die von einer fremden Frau stammt (auch Spenderinnenmilch genannt).

22 Heute existieren weltweit in 18 Ländern Milchbanken. In Deutschland existieren 15 Frauenmilchbanken. 1997 waren es noch 17, die damals insgesamt 12'000 Liter Spenderinnenmilch auf ärztliche Verordnung abgaben; SPRINGer, S. 6. Für weitere Informationen zur Entwicklung und dem aktuellen Stand der Milchbanken siehe Вотн, S. 4 ff.

23 Ausführliche Beschreibung der Funktionsweise einer Frauenmilchbank siehe Both, S. 6 und Springer, passim.

24 Ausführlich zur Hitzebehandlung siehe SPRINGER, S. 30.

25 Z.B. Lagern, Pasteurisieren und Aufbereiten der Milch.

26 Diese sind nicht zu verwechseln mit der in der Praxis als Milchküche bezeichneten Abteilung eines Spitals. Die Milchküche ist mit der Vorbereitung der Flaschennahrung (künstlicher Säuglingsmilch) den Kantonsspitälern Luzern, St. Gallen, Aarau, Bern und Basel Milchbanken betrieben. ${ }^{27}$ Sie sind in der Regel organisatorisch und administrativ den neonatologischen Abteilungen angegliedert.

$\left[\begin{array}{ll}R z & 9\end{array}\right]$ In der Schweiz wird die gespendete Frauenmilch grundsätzlich nur für Frühgeborene verwendet, solange sie sich in Spitalobhut befinden. In der Regel werden Neugeborene mit einem Gewicht von weniger als 1500 Gramm oder einem Gestationsalter ${ }^{28}$ von weniger als 32 Schwangerschaftswochen mit gespendeter, pasteurisierter Frauenmilch versorgt. Die Bedeutung der Milchbanken und der Bedarf an Frauenmilch nehmen kontinuierlich zu. Jährlich steigt die Zahl der zu behandelnden Frühgeborenen an der Grenze zur Lebensfähigkeit. ${ }^{29}$ Diese unterliegen einem erhöhten Risiko, an einer nektrotisierenden Enterokolitis ${ }^{30}$ zu erkranken. ${ }^{31}$ Durch den Einsatz von Frauenmilch wird diesem Risiko entgegengewirkt. ${ }^{32}$

[Rz 10] Seit einiger Zeit ist zudem anerkannt, dass Frauenmilch auch für kranke, operierte und untergewichtige Säuglinge von Nutzen sein kann. Die Ernährung mit Frauenmilch könnte auch für gewisse erwachsene Patienten von Vorteil sein. So sollen etwa die Nebenwirkungen einer Chemotherapie verringert, dass Immunsystem gestärkt und die Verdauung verbessert werden können. Frauenmilch wurde zudem bereits in der Therapie von Verbrennungsopfern, bei Organtransplantationen und bei Menschen mit Augenleiden erfolgreich eingesetzt. ${ }^{33}$ Der Vorrat an gespendeter Frauenmilch reicht in der Schweiz jedoch nicht aus, um die bisherige Anwendung auszuweiten.

\subsection{Rechtliche Fragen}

[Rz 11] Eine rechtliche Auseinandersetzung mit dem Thema der Milchspende und den rechtlichen Rahmenbedingungen für den Betrieb einer Frauenmilchbank fehlt bis anhin. Im Folgenden werden vorab die völker- und verfassungsrechtlichen Vorgaben dargestellt (Ziff. 2). Anschliessend wird die Rechtslage im sozialversicherungsrechtlichen Kontext erläutert (Ziff. 3). Schliesslich geht es darum, die Frauenmilch als solche rechtlich zu qualifizieren und einige Rechtsfragen im Zusammenhang mit dem Betrieb einer Frauenmilchbank aufzugreifen (Ziff. 4).

betraut.

${ }_{27}$ Milchbanken fehlen in der französischen Schweiz und im Tessin gänzlich.

28 Schwangerschaftsdauer respektive der Entwicklungsgrad des Neugeborenen.

29 Die Schweizerische Gesellschaft für Neonatologie Gestationsalter definiert Frühgeborene, welche zwischen der 22. und der 26. SSW (Schwangerschaftswoche) auf die Welt kommen als «Frühgeborene an der Grenze zur Lebensfähigkeit»; Guidelines, Empfehlungen zur Betreuung von Frühgeborenen an der Grenze der Lebensfähigkeit, SwIss SOCIETY OF NEONATOLOGY WwW.neonet.ch/assets/doc/gestationsalter-d.pdf (besucht am 17.7.08)

30 Schwere Darmentzündung des Früh- und Neugeborenen.

31 Vgl. Ball/Wright, S. 874; LuCAS/COLE, S. 1520; BOth, S. 4.

32 Вотн, S. $5 \mathrm{ff}$.

33 Merhav/Wright/Miles/Van Thiel, passim. 
$\left[\begin{array}{ll}R z & 12\end{array}\right]$ Im Zusammenhang mit der Frauenmilchspende können sich zahlreiche weitere Fragen stellen, insbesondere im Bereich der Haftung. Auf entsprechende Ausführungen wird im vorliegenden Beitrag verzichtet.

\section{Gibt es ein Grundrecht auf optimale Ernährung?}

\subsection{Völkerrechtliche Betrachtung}

[Rz 13] Das moderne Völkerrecht regelt längst nicht mehr nur zwischenstaatliche Beziehungen, auch Individuen können durch Völkerrecht unmittelbar betroffen sein. ${ }^{34}$ Eine völkerrechtliche Betrachtung drängt sich deshalb auf.

[Rz 14] Im Zentrum der Betrachtung steht das zu ernährende Kind. Die WHO hat zum Thema der Kinderernährung, insbesondere zur Säuglingsernährung, eine globale Strategie entwickelt. Diverse Dokumente, Studien und Informationen wurden erarbeitet und publiziert. ${ }^{35}$ In diesen Arbeitsdokumenten legt die WHO eine Rangfolge der optimalen Ernährungsformen für Säuglinge fest:

1. Direktes Stillen durch die Mutter;

2. Abgepumpte Milch der eigenen Mutter;

3. Abgepumpte, pasteurisierte Milch einer Spenderin;

4. Formula-Milch (künstliche Säuglingsnahrung). ${ }^{36}$

[Rz 15] Diesen Dokumenten kommt keine Rechtsverbindlichkeit zu, dennoch widerspiegeln sie den wissenschaftlichen Konsens. Zusammen mit dem universellen Prinzip des Kindswohls ${ }^{37}$ können diese Empfehlungen als Leitsätze und Auslegungshilfe für alle kindesspezifischen Ernährungsfragen dienen.

[Rz 16] Laut den Ausführungen derWHO ist das Recht aufNahrung ein Menschenrecht: «Freedom from hunger and malnutrition is a basic human right and their alleviation is a fundamental prerequisite for human and national development. s $^{38}$ Besteht nun - in diesem Sinn - in unserer Rechtsordnung ein Grundrecht auf optimale Ernährung oder gar auf Gesundheit und Gesundheitsförderung? $?^{39}$

34 Z.B. KäLIN/KüNZLI, S. 7.

35 www.who.int/topics/breastfeeding/en/ (besucht am 11.7.07); www. who.int/nutrition/publications/infantfeeding/en/index.html (besucht am 11.7.07); WHO, Global Strategy, passim; siehe auch BrEINING-KAUFMANN, S. 46 .

$36 \mathrm{Vgl}$. Work Group on Breastfeeding, S. 1036; WHO/Unicef, S. 10, Ziff. 18. Eine ausführliche Darstellung über die Initiative der WHO und UNICEF zum Thema Stillen ist bei Hodgkin/NEWELL, S. 356 zu finden.

37 Vgl. Art. 3 Übereinkommen über die Rechte des Kindes (KRK) vom 20. November 1989, SR 0.107.

38 www.who.int/nutrition/en/ (besucht am 11.7.07); ausführlich zum «Recht auf Nahrung» als Menschenrecht Breining-Kaufmann, S. $57 \mathrm{ff.}$

39 Ausführlich zum Thema «Recht auf Gesundheitsversorgung als Menschenrecht» siehe STEINER, S. $116 \mathrm{ff}$. Insbesondere krankheitsbedingte Kindersterblichkeit durch Schwächung des Immunsystems lässt sich
[Rz 17] Eine explizite Erwähnung des Rechts auf Ernährung findet sich in der Verfassung der $\mathrm{WHO}^{40}$ nicht. Vielmehr wird in der Präambel verschiedentlich auf die Gesundheit Bezug genommen oder gar explizit auf die Förderung der Entwicklung des Kindes hingewiesen. Die bekannte Umschreibung der Gesundheit steht am Anfang der Verfassung. ${ }^{41}$ Die Verfassung der WHO begründet indes keine justitiablen Ansprüche, sie ist rein programmatischer Natur. ${ }^{42}$ Die WHO wurde mehrheitlich mit Förderungspflichten ausgestattet. Die Verantwortung für den Gesundheitsschutz bleibt letztlich bei den einzelnen Mitgliedstaaten. ${ }^{43}$

$\left[\begin{array}{ll}R z & 18\end{array}\right]$ Art. 25 der UNO-Menschenrechtserklärung ${ }^{44}$ gilt als grundlegende Norm bezüglich des Rechts auf Gesundheit und Nahrung. Laut dieser Bestimmung hat jeder Mensch «Anspruch auf eine Lebenshaltung, die seine und seiner Familie Gesundheit und Wohlbefinden, einschliesslich Nahrung, Kleidung, Wohnung, ärztliche Betreuung und der notwendigen Leistungen der sozialen Fürsorge, gewährleistet ...». In Absatz 2 wird sodann der Anspruch von Mutter und Kind konkretisiert: «Mutter und Kind haben Anspruch auf besondere Fürsorge und Unterstützung.» Die Schweiz ist seit dem Beitritt zur UNO verpflichtet, deren Konvention und Erklärung zu respektieren. Die UNOMenschenrechtserklärung ist indes kein juristisch verbindliches Dokument. Es handelt sich beim Grundrechtskatalog der UNO lediglich um ein Ideal, das es anzustreben gilt. ${ }^{45}$ Insofern kann Art. 25 der Menschenrechtskonvention allenfalls als Auslegungshilfe beigezogen werden. ${ }^{46}$

$\left[\begin{array}{ll}R z & 19\end{array}\right]$ UNO-Pakt $I^{47}$ und UNO-Pakt $I^{48}$ enthalten hingegen konkretere Anknüpfungspunkte. Allerdings lässt sich v.a. aus den Normen des UNO-Pakt I keine konkrete subjektive

auf unzureichende Ernährung zurückführen. Konsequenterweise liesse sich deshalb das Thema "optimale Ernährung» respektive das Menschenrecht auf Nahrung nur in Beziehung zu anderen Grundrechten (wirtschaftliche, politische und soziale Rechte) beantworten; BrEININGKaUfMANN, S. 46 und 50.

40 Verfassung der Weltgesundheitsorganisation (WHO) vom 22. Juli 1946, SR 0.810.1.

41 Präambel, Verfassung WHO.

42 Z.B. LANDOLT, S. 124

43 Vgl. Art. 118 BV.

44 Offizielle Bezeichnung: «Die Allgemeine Erklärung der Menschenrechte von 1948», deutsche Fassung der Erklärung www.unhchr.ch/udhr/lang/ ger_print.htm (besucht am 11.7.07).

45 www.humanrights.ch/cms/front_content.php?idcat=217 (besucht am 11.7.07); Z.B. Pfiffner Rauber, S. 68.

46 Vgl. Guillod/Sprumont, S. 345. Die UNO-Menschenrechtskonvention gewährt den Menschen mehr als ein Recht, frei von Hunger zu sein. Gemäss dieser Konvention kann ein «Anspruch auf ausreichende Nahrung für Gesundheit und Wohlbefinden» abgeleitet werden, siehe ausführlich dazu Breining-Kaufmann, S. 57 und 58 sowie Kalin/KunzLI, S. 304 ff. Ausreichend Nahrung entspricht aber nicht dem Bedürfnis nach einer optimalen Nahrung.

47 Internationaler Pakt über wirtschaftliche, soziale und kulturelle Rechte vom 16. Dezember 1966, i.K. seit 18. September 1992, SR 0.103.1.

48 Internationaler Pakt über bürgerliche und politische Rechte vom 16. Dezember 1966, i.K. seit 18. September 1992, SR 0.103.2. 
Anspruchsgrundlage im Bereich der Krankheitsbehandlung respektive Gesundheitsförderung ableiten. ${ }^{49}$ Es handelt sich lediglich um Zielvorgaben. In der Lehre finden sich allerdings gegenteilige Meinungen. ${ }^{50}$ Art. 12 UNO-Pakt I postuliert das Recht auf ein Höchstmass an körperlicher und geistiger Gesundheit. ${ }^{51}$ «Die von den Vertragsstaaten zu unternehmenden Schritte zur vollen Verwirklichung dieses Rechts umfassen die erforderlichen Massnahmen zur Senkung (...) der Kindersterblichkeit sowie zur gesunden Entwicklung des Kindes.135 ${ }^{52}$ Bezüglich gesunder Entwicklung der Kinder kann wiederum auf die Ernährungsempfehlung der WHO verwiesen werden: Frauenmilch geniesst Priorität vor Formula-Milch. Der Anspruch auf Versorgung im medizinischen und nahrungstechnischen Bereich geht allerdings nicht über den der schweizerischen Verfassung hinaus. ${ }^{53}$

[Rz 20] Spezifisch für die Gesundheitsbedürfnisse der Kinder wurde die sogenannte Kinderrechtskonvention geschaffen. Der von der Schweiz vorbehaltlos ratifizierte Art. 24 der Kinderrechtskonvention ist im Bereich der Gesundheitsförderung von Bedeutung. Gefordert wird wiederum ein Erreichen des Höchstmasses an Gesundheit. Weiter wird eine Reduktion der Säuglings- und Kindersterblichkeit postuliert. Für die hier behandelten Fragen ist vor allem von Interesse, dass in derselben Bestimmung die Bekämpfung von Fehlernährung gefordert und

49 Vgl. z.B. BGE 125 III 277, E. 2d; BGE 121 V 246, E. 2b; Nowak, S. 9.

50 Z.B. KünzLI, S. 537; vgl. auch Ausführungen des UN-Ausschusses zur Rechtsnatur des Paktes zitiert nach KäLIN/KünzLI, S. 303; siehe weiter die allgemeinen Bemerkungen des Ausschusses für wirtschaftliche, soziale und kulturelle Rechte zum Pakt I in KäLin/MaLINVERNI/NowAK, S. 304.

51 Zum Thema Recht auf Gesundheit und Art. 12 UNO-Pakt I siehe Künzli/ Kälin, S. 141 ff. Ebenfalls von Interesse bezüglich Ernährung ist Art. 11 UNO-Pakt I. Aus diesem Artikel lässt sich die grundlegende Erkenntnis ableiten, «dass das Recht auf Nahrung Voraussetzung sämtlicher Menschenrechte ist und eines der Grundelemente für die Verwirklichung der Menschenwürde bildet.»; Breining-Kaufmann, S. 59. Das Recht auf Nahrung lässt sich in zwei Teile gliedern; erstens dem Recht auf ausreichende Ernährung und zweitens dem Schutz vor Hunger. Schutz vor Hunger meint in erster Linie, das zum Überleben erforderliche Mindestmass an Kalorien zu erhalten. Das Recht auf ausreichende Ernährung geht weiter und umfasst die genügende Nahrungszufuhr, um ein normales Leben zu führen. Das Recht auf ausreichende Nahrung kann eingeschränkt werden, während der Schutz vor Hunger zum absolut geschützten Kerngehalt von Art. 11 gehört. Weiter lässt sich aus dem Recht auf Leben (Art. 6 UNO Pakt I, siehe auch Art. 2 Konvention vom 4. Novenmber 1950 zum Schutze der Menschenrechte und Grundfreiheiten (EMRK), SR 0.101) ein Schutz vor Hunger ableiten. Dieser Minimalstandard wird wiederum als Voraussetzung angesehen, um alle anderen Menschenrechte wahrnehmen zu können; BreInING-KAUfMAnN, S. $59 \mathrm{ff}$.

52 Art. 12 Abs. 2 lit. a UNO-Pakt I.

53 Paiffner Rauber, S. 71. Zu erwähnen ist, dass sich unter dem Dach der UNO weitere Organisationen mit entsprechenden Erklärungen finden lassen, welche sich explizit mit dem Thema Nahrung befassen. Zu denken ist etwa an die World Food Conference und die "Universal Declaration on the Eradication of Hunger and Malnutrition». Weiteren Statuten internationaler Organisationen sind ebenfalls diverse Bekenntnisse zum Recht auf Nahrung zu entnehmen; BREINING-KaUfMANN, S. 96. die Vermittlung der Vorteile des Stillens postuliert werden. ${ }^{54}$ Die Kinderrechtskonvention stellt diesbezüglich für das einzelne Neugeborene aber keine direkte Anspruchsgrundlage dar, sie hat lediglich programmatischen Charakter. ${ }^{55}$

[Rz 21] Auf europäischer Ebene finden sich - neben der bereits erwähnten EMRK - keine Grundlagen, die im behandelten Bereich von spezifischem Interesse sein könnten. ${ }^{56}$

[Rz 22] Abschliessend lässt sich festhalten, dass bezüglich der Ernährung ein gewisser internationaler Schutz besteht, dieser Schutz jedoch abgestuft ist. In diesem Sinne kann zwischen dem Schutz vor Hunger, dem Recht auf ausreichende Nahrung und letztlich dem hier in Frage stehenden Recht auf optimale Ernährung unterschieden werden. In keiner der behandelten Rechtsquellen wird ein einklagbarer Rechtsanspruch auf optimale Ernährung postuliert. ${ }^{57}$ Das Bestreben, allen Menschen - insbesondere den Kindern - eine optimale Ernährung zu gewährleisten, kann jedoch auf internationaler Ebene durchaus als Ideal und somit als Zielvorgabe angesehen werden. Dabei gehen die internationalen Quellen, soweit sie dazu Äusserungen enthalten, von der Ernährung durch Muttermilch als Optimum aus. Zudem wird in Empfehlungen der WHO die Ernährung mit pasteurisierter Frauenmilch der Ernährung mit Formula-Milch vorgezogen. $^{58}$

\subsection{Grundlagen in der schweizerischen Bundesver- fassung}

[Rz 23] In der Schweiz herrscht allgemein eine zurückhaltende Praxis im Bereich der sozialen Grundrechte. Dies zeigt sich unter anderem daran, dass lediglich aus Art. 12 BV 59 (Recht auf Hilfe in Notlagen) und Art. 19 BV (Anspruch auf Grundschulunterricht) justitiable grundrechtliche Ansprüche abgeleitet werden können. Der Umfang der Ansprüche wird in der Praxis zudem auf ein Minimum beschränkt. ${ }^{60}$

[Rz 24] Art. 41 BV postuliert als Sozialziel, dass «jede Person die für ihreGesundheitnotwendigePflegeerhält». EinLeistungsanspruch des Einzelnen ist daraus nicht abzuleiten. ${ }^{61}$ Auch Art. 10 BV verbürgt nach einhelliger Meinung nicht mehr als einen Anspruch

54 Ausführlich zum Inhalt von Art. 24 KRK siehe Hodgkin/NEwELL, S. 343 ff.

55 Botschaft betreffend den Beitritt der Schweiz zum Übereinkommen von 1989 über die Rechte des Kindes vom 29. Juni 1994 (BBI 1994 V, S. 2 ff.), S. $51 \mathrm{ff}$.

$56 \mathrm{Vgl}$. aber betreffend das Recht auf Schutz vor Hunger z.B. Art. 2 EMRK, siehe Fussnote 51.

57 Breining-Kaufmann, S. $57 \mathrm{ff}$.

58 Siehe Fussnote 36.

59 Bundesverfassung der Schweizerischen Eidgenossenschaft vom 18. April 1999, SR 101.

60 GÄCHTER, Soziale Grundrechte, S. 139. Zum Leistungsumfang und der Subsidiarität der Hilfe im Rahmen von Art. 12 BV siehe insbesondere die Ausführungen von Waldmann, S. 355 ff.; siehe als Beleg für die Zurückhaltung der Praxis etwa Urteil 2P.276/2005 vom 7. Mai 2007, E. 3 ff. (Umfang von Art. $19 \mathrm{BV}$ )

61 Vgl. Art. 41 Abs. 4 BV; Häfelin/Haller, N. 215. 
auf medizinische Behandlung in Notfallsituationen, wobei dieser Anspruch in der Systematik der Bundesverfassung eher bei Art. 12 BV anzusiedeln ist. ${ }^{62}$ Im Umfang der elementaren menschlichen Bedürfnisse wie Nahrung, Kleidung und Obdach statuiert Art. 12 BV einklagbare subjektive Ansprüche. Nach einhelliger Meinung begründet das Recht auf Hilfe in Notlagen auch einen Anspruch auf medizinische Betreuung im Rahmen elementarer medizinischer Grundversorgung. ${ }^{63}$ Auf jeden Fall muss ein moderner Staat die auf seinem Gebiete befindlichen Personen nötigenfalls vor dem physischen Verderben bewahren. ${ }^{64}$ Auf Art. $12 \mathrm{BV}$ ist subsidiär abzustellen, wenn das einfache Gesetzesrecht im Ergebnis nicht dem verfassungsrechtlichen Minimalanspruch genügen kann. ${ }^{65}$ Unterlassene medizinische Hilfe in Notlagen im Sinn von Art. 12 BV sowie die Verweigerung von Nahrung, die für das menschenwürdige Überlegen notwendig ist, kann demnach eine Grundrechtsverletzung darstellen. Die Versorgung mit Frauenmilch dürfte indes kaum in den Geltungsbereich dieses Grundrechtesfallen, da diesefür das Überleben nicht unabdingbar ist und durch künstliche Milch substituiert werden kann.

[Rz 25] Art. 11 BV statuiert den expliziten Schutz der Kinder: "Kinder (...) haben Anspruch auf besonderen Schutz ihrer Unversehrtheit und auf Förderung ihrer Entwicklung.» Der genaue Bedeutungsgehalt dieser neu in die Bundesverfassung aufgenommenen Norm ist teilweise umstritten. ${ }^{66}$ Auf den ersten Blick ist man geneigt, in Art. 11 ein neues Sozialrecht zu erkennen. ${ }^{67}$ Allerdings ist der Gesetzeswortlaut derart vage formuliert und auslegungsbedürftig, dass diese Norm eher als programmatisch denn als anspruchsbegründend zu verstehen ist. Eine abschliessende Beurteilung musste das Bundesgericht noch nicht vornehmen. ${ }^{68}$ Art. $11 \mathrm{BV}$ ist in diesem Sinne als politisches Zeichen zu Gunsten der Jugend zu verstehen. ${ }^{69}$

\subsection{Zwischenfazit}

[Rz 26] Zusammenfassend lässt sich festhalten, dass auf Grund der geltenden verfassungs- und völkerrechtlicher Vorgaben für den Säugling kein justitiabler Anspruch auf optimale Ernährung in Form von Frauenmilch besteht. Erkennbar wird aber immerhin eine deutliche Präferenz für die Ernährung der Säuglinge mit Muttermilch und subsidiär mit Frauenmilch. Gefordert ist damit

62 GÄCHTER, Grenzen der Solidarität, S. 485

63 Coullery, Grundrechtsanspruch auf med. Leistungen, S. 633; GÄCHTER, Grenzen der Solidarität, S. 483, mit weiteren Hinweisen.

64 Siehe etwa BGE 131 | 166, E. 8.2.

65 BGE 121 । 367, E. 2b, 2c

66 KaYSER/Richter, S. 1029.

67 GI.M. RHINOW, S. 220.

68 BGE 126 || 377, E. 5c; BGE 129 | 12, E. 10.5.3; Urteil 2P.324/2001 vom 28 März 2002; Urteil 6S.361/2002 vom 5. Juni 2003; Rhinow, S. 220; Häfelin/ Haller, N. 291, N. 910.

69 Aubert/Mahon, Art. 11 N. 2. Siehe insbesondere Votum Koller, Amtliches Bulletin des Nationalrates 1998, Sonderausgabe Verfassungsreform, S. $191 \mathrm{ff}$. der Gesetzgeber, der für das Stillen sowie die Versorgung mit Frauenmilch günstige Voraussetzungen schaffen sollte.

\section{Sozialversicherungsrechtliche Regelungen}

[Rz 27] Obwohl ein subjektiver Anspruch auf optimale Ernährung aus grundrechtlicher Sicht zu verneinen ist, wird in der Praxis die Ernährung der Frühgeborenen mit Frauenmilch praktiziert. Wer kommt aber für die entsprechenden Kosten auf? Die Antworten müssen im Bundessozialversicherungsrecht gesucht werden. Vorliegend stehen die Rechtsverhältnisse zwischen der Sozialversicherung und der Mutter (respektive dem Säugling) und jene zwischen der Sozialversicherung und der Spenderin im Vordergrund.

\subsection{Kostenübernahme für den Konsum von Frauen- milch}

[ Rz 28] Die gespendete Milch wird in der Regel an Kinder im Spital abgegeben. Die Betriebskosten der Milchbanken werden meistens durch die neonatologischen Abteilungen getragen. Somit werden diese einerseits durch allfällige Beiträge der öffentlichen Hand an die Spitalbetriebskosten und anderseits durch Tagespauschalen für den Spitalaufenthalt durch die entsprechende Sozialversicherung finanziert. ${ }^{70}$

[Rz29]DieKostenfürPflegeundAufenthaltgesunderNeugeborener sind durch die obligatorische Krankenpflegeversicherung der Mutter gedeckt. ${ }^{71}$ Bei den Konsumenten der Frauenmilchbank handelt es sich aber überwiegend um kranke oder frühgeborene Säuglinge. Für die Kosten des kranken Neugeborenen wiederum kommt grundsätzlich - soweit kein Geburtsgebrechen vorliegt die Krankenversicherung der Neugeborenen auf.

[Rz 30] Die Kosten aller notwendigen medizinischen Massnahmen bei Minderjährigen im Rahmen von Geburtsgebrechen fallen in den Anwendungsbereich der Invalidenversicherung (Art. $13\left(\mathrm{IG}^{72}\right)$, welche in diesem Bereich gewissermassen zu einer Krankenversicherung wird, ${ }^{73}$ da es sich bei den Geburtsgebrechen auch um Krankheiten im Rechtssinn handelt (Art. 3 Abs. 2 ATSG $^{74}$ ). Die Besonderheit der Geburtsgebrechen besteht darin, dass sie bereits bei vollendeter Geburt bestehen. ${ }^{75}$ Die anerkannten Geburtsgebrechen sind in der Geburtsgebrechenverordnung ${ }^{76}$ abschliessend aufgeführt. Frühgeborene mit einem

70 Siehe z.B. Art. 49 KVG

7 Art. 29 Abs. 2 lit. d des Bundesgesetz über die Krankenversicherung (KVG) vom 18. März 1994, SR 832.10.

72 Bundesgesetz über die Invalidenversicherung (IVG) vom 19. Juni 1959, SR 831.20.

73 MAURER, S. 153.

74 Bundesgesetz über den Allgemeinen Teil des Sozialversicherungsrechts (ATSG) vom 6. Oktober 2000, SR 830.1.

75 Art. 3 Abs. 2 ATSG.

76 Verordnung über Geburtsgebrechen (GgV) vom 9. Dezember 1985, SR 831.232.21. 
Geburtsgewicht von unter 2000 Gramm gelten gemäss dieser Verordnung bis zum Erreichen von 3000 Gramm als an einem Geburtsgebrechen leidend. ${ }^{77}$ Dielnvalidenversicherung deckt die Kosten für medizinische Massnahmen, welche zur Behandlung der durch die Geburtsgebrechenverordnung anerkannten Geburtsgebrechen notwendig sind. Erfasst sind unter anderem medizinische Untersuchungen, ambulante, teilambulante oder stationäre Pflege, Analysen, Arzneimittel und sonstige Therapien. Im Gegensatz zur Krankenversicherung kennt die Invalidenversicherung keine Kostenbeteiligung, ${ }^{78}$ wodurch diejenigen, die Leistungen für Geburtsgebrechen beziehen, gegenüber den Leistungsempfängern der Krankenversicherung leicht privilegiert werden.

[ Rz 31] Unabhängig davon, welche Sozialversicherung dafür aufzukommen hat, werden die Kosten für den Konsum von Frauenmilch nicht separat verrechnet. Eine zusätzliche Vergütung würde sich lediglich rechtfertigen, wenn die Kosten der Frauenmilchbanken sehr viel höher wären als die Kosten für eine Ernährung mit herkömmlicher, industriell gefertigter Säuglingsmilch. ${ }^{79}$ Dies ist jedoch nicht der Fall.

[Rz 32] Eine weitere - gegenwärtig allerdings hypothetische Fragestellung läge bei der Kostenübernahme für die ambulante Versorgung mit Frauenmilch. Diese Form der Ernährung wird heute noch nicht praktiziert, da die vorhandenen Milchreserven kaum ausreichen, um den Tagesbedarf für stationär behandelte Kinder zu decken.

\subsection{Mietkosten für Abpumpgeräte}

[Rz 33] Nebst den Betriebskosten für die Milchbanken entstehen noch weitere Kosten. In diesem Zusammenhang sind die Kosten für die Milchpumpen von Interesse, die von den Spenderinnen benützt werden. Abgepumpt werden kann mit Hilfe eines manuellen oder elektrischen Abpumpgerätes. Auf dem Markt werden diverse Modelle mit verschiedenen Betriebsvarianten angeboten. Eine Spenderin wird langfristig und regelmässig abpumpen wollen; sei dies um das eigene Kind zu versorgen, zusätzliche Milch spenden zu können, um einen Milchstau zu verhindern oder um den Milchfluss anzuregen. Sie ist deshalb auf ein professionelles und qualitativ hochstehendes Gerät angewiesen. Die Geräte sind in der Anschaffung relativ teuer und werden nur für eine bestimmte Zeitspanne gebraucht. Ein Kauf lohnt sich demnach kaum. Die Geräte werden deshalb meist zur Miete bezogen. Entweder wird das entsprechende Modell direkt vom Spital zur Verfügung gestellt oder die Spenderin kann mit einem ärztlichen Zeugnis ein solches Gerät beziehen.

77 Vgl. GgV, Anhang XX, Ziff. 494. Die Frühgeborenen mit einem Geburtsgewicht von mehr als 2000 Gramm leiden in den meisten Fällen an einem anderen Leiden (z.B. an einer neonatalen Infektion), das von der Geburtsgebrechenverordnung ebenfalls erfasst ist. Deshalb fallen auch sie meist in den Geltungsbereich der IV.

78 Siehe Art. 64 KVG.

79 Vgl. Art. 49 Abs. 2 KVG.
[Rz 34] Das Risiko der Mutterschaft erfasst die Schwangerschaft, die Niederkunft und die darauf folgende Erholungszeit der Mutter und gilt grundsätzlich nicht als Krankheit. ${ }^{80}$ Die besonderen Leistungen der Mutterschaft werden nebst den gleichen Leistungen wie bei Krankheit von der obligatorischen Krankenpflegeversicherung übernommen, ${ }^{81}$ wobei eine Besonderheit der Leistungen bei Mutterschaft darin besteht, dass dieVersichertekeineKostenbeteiligung in Form von Franchise und Selbstbehalt zu tragen hat. ${ }^{82}$ Für die Spenderin ist es deshalb nicht unerheblich, ob die Miete für die Milchpumpe unter Mutterschaft oder unter Krankheit subsumiert wird. Nach der Systematik des KVG handelt es sich bei der Miete für eine Milchpumpe um eine Leistung für Krankheit, nicht für Mutterschaft. ${ }^{83}$

[Rz 35] Bei der Miete einer Milchpumpe handelt es sich nicht um eine medizinische Leistung im eigentlichen Sinne, sondern um das Entgelt für die Leihe eines Geräts. Das KVG kennt den Begriff der Hilfsmittel, wie er im IVG ${ }^{84}$ oder UVG ${ }^{85}$ anzutreffen ist, nicht. Hingegen kennt das KVG kassenpflichtige Mittel und Gegenstände, welche abschliessend im Anhang 2 der KLV ${ }^{86}$ aufgeführt werden. ${ }^{87}$ Mittel und Gegenstände müssen «diagnostischen, therapeutischen oder pflegerischen Zielsetzungen» dienen, d.h. sie bezwecken «hauptsächlich die Gesundheit wiederherzustellen, Schmerzen zu lindern oder eine drohende Verschlechterung des Gesundheitszustandes zu verhindern ${ }^{88}$ In diesem Sinne müssen zwei Voraussetzungen gegeben sein: Einerseits muss die Verwendung der Milchpumpen durch den Arzt vorgeschrieben werden und anderseits soll die Anwendung der Pumpe einen gewissen Zweck erfüllen. Im hier behandelten Fall wird durch das Abpumpen der überschüssigen Milch allenfalls ein Milchstau bei der Spenderin verhindert und damit einer Verschlechterung des Gesundheitszustandes vorgebeugt, anderseits wird die Gesundheit des Konsumenten gefördert.

[Rz 36] Das EDI hat die Miete der Pumpe auf die Mittelund Gegenständeliste (MiGeL) aufgenommen. Die Krankenversicherung ist nicht befugt, mehr als den aufgelisteten Betrag (CHF 2.-pro Tag) zu vergüten. Der in der MiGeL aufgeführte Höchstvergütungsbetrag entspricht in der Regel einem Durchschnittspreis der auf dem Markt erhältlichen Produkte. ${ }^{89}$ In

80 Art. 5 ATSG.

81 Art. 29 Abs. 1 KVG.

82 Art. 64 Abs. 7 KVG.

${ }^{83}$ Die Vergütung der Milchpumpen ist nicht im Leistungskatalog der Mutterschaft aufgeführt (Art. 29 KVG und Art. 13-16 KLV).

${ }^{84}$ Art. 21 f. IVG

85 Art. 11 des Bundesgesetzes über die Unfallversicherung (UVG) vom 20. März 1981, SR 832.20.

86 Verordnung über Leistungen in der obligatorischen Krankenpflegeversicherung (KLV) vom 29. September 1995, SR 832.112.31.

${ }^{87}$ Die sogenannte Mittel- und Gegenständeliste (MiGeL) kann auf der Homepage des BAG abgerufen werden (besucht am 11.7.07).

${ }_{88} \mathrm{Vgl.} \mathrm{BGE} 109 \mathrm{~V}$ 167, E. $3 \mathrm{ff}$

89 Ziff. $2.2 \mathrm{MiGeL}$ 
der Praxis sind die effektiven Mietkosten für die Pumpmaschine jedoch höher. Für die gängigsten Geräte, die meist von den Spitälern ausgewählt werden und als optimal gelten, belaufen sie sich aufca.CHF3.40 proTag. DieSpenderin wird damit verpflichtet, die Differenz zwischen der Miete und der Kostendeckung selbst zu tragen. Hier sollte ein Ausgleich geschaffen werden. Entweder muss die Spenderin die Möglichkeit haben, auf ein günstigeres Gerät umzusteigen, ${ }^{90}$ wobei die Qualität der Pumpe ausreichen muss, um den Willen zur regelmässigen Spende zu begünstigen. Anderseits könnten, wie dies in der Praxis häufig praktiziert wird, die Maschinen direkt vom Spital zur Verfügung gestellt werden. Die Kosten für Miete oder Anschaffung würden in diesem Falle vom Spital getragen und letztlich wiederum über die Tagespauschale abgegolten. Anzumerken bleibt, dass im Rahmen der Bundessozialversicherungen grundsätzlich kein Anspruch auf die optimalsten Hilfsmittel resp. Mittel und Gegenstände besteht. Der Anspruch erstreckt sich in der Regel auf einfache und zweckmässige Leistungen.91

[Rz 37] Zusammenfassend lässt sich sagen, dass die Miete der Milchpumpe grundsätzlich von der Krankenversicherung der Spenderin getragen wird. Die Spenderin vollzieht die vor allem zeitlichen Aufwendungen der Milchspende ohne finanzielle Abgeltung. Gleichzeitig muss sie aber mit negativen finanziellen Folgen rechnen (Kostenbeteiligung, allfällige Tragung eines Differenzbetrags zwischen vergütetem Betrag und effektiven Mietkosten), was der Bereitschaft zur Milchspende sicherlich nicht zuträglich ist.

\section{Die juristische Qualifikation der gespendeten} Milch und die rechtlichen Grundlagen für den Betrieb von Milchbanken

\subsection{Sachenrechtliche Qualifikation der Frau- enmilch}

[Rz 38] In der Schweiz wird die Frauenmilch gegenwärtig nur spitalintern verwendet. Zukunftsszenarien wie der Austausch der Frauenmilch unter den Spitälern oder der Handel mit Frauenmilch ziehen sachen- und obligationenrechtliche Fragen nach sich. Im Hinblick auf die Verkehrsfähigkeit sowie auf handels- und vermögensrechtliche Fragen im Zusammenhang mit der Frauenmilch bildet deren sachenrechtliche Qualifikation die Grundlage für eine weiterführende Diskussion.

[Rz 39] Die Lehre hat den Begriff der Sache im Rahmen des $\mathrm{ZGB}^{92}$ als unpersönlichen, körperlichen, für sich bestehenden Gegenstand, der der menschlichen Herrschaft unterworfen

\footnotetext{
90 Dies ist auf Grund der Austauschbefugnis im Bereich der MiGeL auch möglich.

91 Vgl. Art. 21 Abs. 2 IVG; als Beispiel BGE 130 V 163.

92 Schweizerisches Zivilgesetzbuch vom 10. Dezember 1907, SR 210.
}

werden kann, definiert. ${ }^{93}$ Die vier Begriffsmerkmale werden schon seit der Entstehung des ZGB verwendet, der generellabstrakte Sachbegriff wurde seither nicht mehr modifiziert. Gerade die Entwicklungen der Medizin, insbesondere in der Transplantations- und Fortpflanzungsmedizin, werfen immer wieder neue Fragen zum Sachbegriff auf. ${ }^{94}$

[Rz 40] Vor allem die Auslegung des Begriffmerkmals der Unpersönlichkeit bereitet Schwierigkeiten: Grundsätzlich gilt jede Sache, die vom Menschen verschieden ist, als unpersönlich. ${ }^{95}$ Das als Abgrenzung zwischen Person und Sache verstandene Begriffsmerkmal ist Ausfluss ethischer und moralischer Überlegungen. ${ }^{96}$ Vorwiegend auf Grund der fehlenden Unpersönlichkeit sind weder der menschliche Körper noch der menschliche Leichnam Sachen im Rechtssinn. ${ }^{97}$ Es können somit daran keine sachenrechtlichen Herrschaftsrechte begründet werden. ${ }^{98}$ Auch alle mit dem menschlichen Körper verbundenen Organe, Körperteile oder festverbundenen Hilfsmittel ${ }^{99}$ gelten nicht als Sachen im Rechtssinn. ${ }^{100}$ Erst mit der Trennung vom Körper entsteht die Sacheigenschaft. ${ }^{10}$

[Rz 41] Mit dem Inkrafttreten des Transplantationsgesetzes ${ }^{102}$ bestehen seit 1. Juli 2007 auf Bundesebene Legaldefinitionen von Organen, Geweben und Zellen. ${ }^{103}$ Frauenmilch fält nicht unter diese Definitionen. Es handelt sich bei ihr vielmehr um ein menschliches Sekret. Im medizinischen Sinn ist ein Sekret ein durchDrüsenzellenabgesondertesProduktmitbiologischaktiven Stoffen. Sachenrechtlich ist es jedoch nicht entscheidend, ob es sich beim zu qualifizierenden Gebilde um einen Körperteil, um ein Organ, um Gewebe, um Zellen oder eben um ein Sekret handelt. Vielmehr greift die Unterscheidung erst später, nämlich bei der Umschreibung der Verkehrsfähigkeit. Bei Organen beispielsweise wird diese rechtlich eingeschränkt; eine Kommerzialisierung der Spende ist nicht zugelassen. ${ }^{104}$

[Rz 42] Das Sekret erfüllt, genau wie ein vom menschlichen Körper getrennter Körperteil oder ein abgetrenntes Organ, alle vier Begriffsvoraussetzungen der Sache. ${ }^{105}$ Denkbar wäre allenfalls,

\footnotetext{
93 Rey, N. 66; Tuor/Schnyder/SChmid, S. 730; Wiegand, N. 6.

$94 \mathrm{Vgl.} \mathrm{KäLIN,} \mathrm{S.} 140 \mathrm{ff}$.

95 Schmid/Hürlimann-Kaup, N. 3 ff.; Kălin S. 61.

96 REY, N. 101 ff.; KÄLIN, S. 60.

$97 \mathrm{H}_{A A B}$, S. 11, WIEGAND, N. 18

98 Schmidt/Hürlimann-Kaup N. 3 ff.; Nach Ablauf einer gewissen Zeit kann der Leichnam als Sache bezeichnet werden, REY, N. 112; WIEGAND, N. 19. Anderer Meinung KäLIN, S. 68.

99 Z.B. Herzschrittmacher, künstliches Hüftgelenk.

100 KäLIN, S. 106; REY, N. 109 ff.

101 REY, N. 106; KälIN, S. 98; WiEgAnd, N. 18; Für Deutschland vgl. Schreiber, N. 9; Es finden sich keine Ausführungen zu dieser Thematik im Botschaftstext des Transplantationsgesetzes.

102. Bundesgesetz über die Transplantation von Organen, Geweben und Zellen vom 8. Oktober 2004, SR 810.21.

${ }^{103}$ Art. 3 lit. a-c Transplantationsgesetz.

104 Art. 6 Transplantationsgesetz; KäLIN, S. 87; REY, N. 195a.

${ }^{105}$ Es macht keinen Sinn, die Sacheigenschaft bei Sekreten zu negieren
} 
die im Zusammenhang mit dem Fortschritt der Forschung und der Medizin auftauchenden rechtlichen Fragen, insbesondere mit Bezug auf das Eigentum und die Verkehrsfähigkeit, durch besondere Bestimmungen zu lösen. Für die Frauenmilch allein rechtfertigen sich solche Überlegung allerdings nicht.. ${ }^{106}$ Aus der Qualifikation der abgepumpten Frauenmilch als Sache folgt, dass an ihr auch Eigentum begründet werden kann. ${ }^{107}$ Dieses geht mit dem Abpumpprozess automatisch an die Frau über. ${ }^{108}$ Erst mit vollzogener Spende, d.h. der Ablieferung bei der Milchbank, geht das Eigentum auf das Spital über.

[ $R z$ 43] Die Spende erfolgt in der Praxis in der Regel unentgeltlich. ${ }^{109}$ De lege ferenda liegt es aber am Gesetzgeber zu entscheiden, ob bei der Frauenmilchspende wie bei der Organspende das altruistische Element und der Schutz der Spenderinnen vor "Selbstausbeutung» im Vordergrund stehen und die Verkehrsfähigkeit beschränktwerden soll oder nicht. ${ }^{110} \mathrm{Am}$ ehesten könnte man sich eine mit der Blutspende vergleichbare Regelung vorstellen, wo die medizinischen Schutzstandards im Vordergrund stehen ${ }^{111}$ und die Praxis auf der Freiwilligkeit der Spende aufbaut.

\subsection{Frauenmilch als Arznei- oder als Lebensmittel?}

[Rz 44] In den USA wird aus Frauenmilch bereits kommerzieller Nutzen gezogen. ${ }^{12}$ In einigen Staaten wird die Frauenmilch als Arzneimittel gehandelt, in anderen wird sie als Lebensmittel deklariert und schliesslich bezeichnen sie gewisse Staaten als menschliches Sekret. ${ }^{113}$ Gerade in Bezug auf den Schutz des

und damit die nachvollziehbare und sinnvolle Qualifizierung der abgetrennten Körperteile und Organe als Sache ebenfalls in Frage zu stellen.

${ }^{106}$ Anders sieht die Sache in Bezug auf die Schutzbedürftigkeit von menschlichen Embryonen und Keimgut aus. Bei diesen ist die Sacheigenschaft umstritten, siehe z.B. KäLIN S. 140 ff.; REY, N. 103; WIEGAND, N. 18.

107 Pelet, S. 178.

108 Dies im Einklang mit der herrschenden Lehre, siehe REY, N. 107; WIEGAND, N. 18; a.M. KäLıN S. 104. Die Frage wurde in den siebziger Jahren des letzten Jahrhunderts ebenfalls noch kontrovers diskutiert, vgl. dazu $\mathrm{H}_{A A B}$, S. 11.

${ }^{109}$ Die Abgeltung des Aufwandes ist jedoch üblich. Ausführlicher zur Verkehrsfähigkeit und Eigentumsfähigkeit Kälin, S. 105. Vgl. auch Art. 4 Bundesgesetz über die Forschung an embryonalen Stammzellen (StFG) vom 19. Dezember 2003, SR 810.31, Art. 6 Transplantationsgesetz, Art. 21 Bundesgesetz über die medizinisch unterstützte Fortpflanzung (FMedG) vom 18. Dezember 1998, SR 810.11.

110 Zur ethischen Betrachtung des Handelsverbotes mit Organen siehe z.B. Ach/ANDERHEIDEn/Quante, S. $189 \mathrm{ff}$. Eine juristische Auseinandersetzung mit dem Handelsverbot ist Z.B. in ZILLGENS, S. $237 \mathrm{ff}$. zu finden.

111 Vgl. Art. 34 ff. des Bundesgesetzes über Arzneimittel und Medizinprodukte (HMG) vom 15. Dezember 2000, SR 812.21

$112 \mathrm{Vgl}$. www.prolacta.com/products.html (besucht 11.7.07).

113 Die FDA (Food and Drug Administration) hat die Frauenmilch als Lebensmittel zugelassen www.prolacta.com/p\&c_faqs.html\#6 (besucht am 11.7.07). Zur allgemeinen Rechtslage in den USA betreffend Stillen siehe www.lalecheleague.org/Illeaderweb/LV/LVJunJul05p51.html
Säuglings ist es von Interesse zu wissen, welchen Kontroll- und Bewilligungsmechanismen ein Anbieter von Frauenmilch unterliegt: Je nach Qualifikation der Frauenmilch greift eine andere Gesetzgebung.

[Rz 45] Mit der medizinischen Qualifikation der Frauenmilch als Sekret ist die Frage noch nicht beantwortet, welchen Normkomplexen die Substanz, die besonders empfindlichen Menschen in einer medizinisch heiklen Situation verabreicht wird, unterliegt. Die Abgrenzung zwischen Arznei- und Lebensmitteln führt denn auch immer wieder zu Schwierigkeiten.

[Rz 46] Als Arzneimittel gilt gemäss Art. 4 Abs. 2 lit. a HMG ${ }^{114}$ ein Produkt chemischen oder biologischen Ursprungs, das zur medizinischen Einwirkung auf den menschlichen oder tierischen Organismus bestimmt ist oder angepriesen wird, insbesondere zur Erkennung, Verhütung oder Behandlung von Krankheiten, Verletzungen und Behinderungen. Frauenmilch wird gegenwärtig zwar in medizinisch heiklen Situationen bei Frühgeborenen angewendet, dient in diesen Fällen objektiv aber nicht der medizinischen Einwirkung auf diese. Vielmehr handelt es sich um eine besonders geeignete Ernährung, die positive medizinische Nebeneffekte aufweist. Massgebend ist jedoch nicht nur die objektive Bestimmung eines Produkts, sondern auch dessen Anpreisung als Arzneimittel. Das Bundesgericht hielt dazu in seinem leading case «Milchkuh Lovely» fest, dass ein Lebensmittel nicht mit «krankheitsvorbeugender, behandelnder oder heilender Wirkung» angepriesen werden dürfe, da es sonst als Heilmittel gelte und die damit verbundenen (strengeren) Vorschriften erfüllen müsse. ${ }^{115}$

[Rz 47] Wenn ein Produkt nicht das vom Heilmittelrecht vorgeschriebene Verfahren durchläuft und nicht als Heilmittel auf den Markt gebracht wird, gelten die Vorschriften des Lebensmittelrechts, «einschliesslich des Verbots, diesem Eigenschaften zur Vorbeugung, Behandlung oder Heilung einer menschlichen Krankheit zuzuschreiben». ${ }^{16}$ Zulässig ist es jedoch, gesundheitsbezogene Werbung über ein Lebensmittel zu lancieren. ${ }^{177}$ Das Bundesgericht hielt weiter fest, das Verbot der krankheitsbezogenen Werbung gelte auch für Nahrungsmittel mit besonderer Zweckbestimmung und für Speziallebensmittel mit ernährungsphysiologischer Wirkung. Bei diesen Nahrungsmitteln liegt der Bezug zum pathologischen Zustand zwar in der Natur der Sache, dennoch darf auch innen nicht der Anschein eines Heilmittels gegeben werden. ${ }^{118}$ Es ist mit anderen Worten

(besucht am 11.7.07).

114 Bundesgesetz über Arzneimittel und Medizinprodukte vom 15. Dezember 2000, SR 812.21

115 BGE 127 I| 91, Regeste. Vgl. weiter zur Abgrenzung HeilmittelLebensmittel Urteil 2A.565/2000 vom 8. Mai 2001.

${ }^{116}$ BGE 127 II 91, E. 3a. aa.; vgl. den neuen Entscheid betreffend gesundheitsfördernde oder krankheitsvorbeugende Wirkung von Gebrauchsgegenständen mit kariesverhütender Eigenschaft: BGE 2A.213/2006

117 BGE 127 ॥ 91, E. 4b.

118 BGE $127 \| 91$, E. 4c. bb. 
dem Anbieter eines Produktes überlassen, ob er dieses als Lebensmittel oder als Heilmittel vertreibt. Massgeblich sind letztlich der Auftritt und die Anpreisung auf dem Markt." ${ }^{119}$

[Rz 48] Das schweizerische Lebensmittelrecht erfasst das Herstellen, Behandeln, Lagern, Transportieren und Abgeben sowie die Kennzeichnung, Anpreisung, Einfuhr und Ausfuhr von Lebensmitteln und Gebrauchsgegenständen. ${ }^{120}$ Die relevante Gesetzgebung wurde anfangs 2006 im Rahmen eines autonomen Nachzuges an die europäischen Bestimmungen zur Lebensmittelhygiene angepasst. ${ }^{121}$ Als Lebensmittel gelten sowohl Nahrungs- als auch Genussmittel. Nahrungsmittel dienen dabei nach der gesetzlichen Definition dem Aufbau oder dem Unterhalt des menschlichen Körpers. ${ }^{122}$ Muttermilch bzw. Frauenmilch dient zweifellos diesem Aufbau und könnte deshalb grundsätzlich als Lebensmittel betrachtet werden.

[Rz 49] Die Generalklausel reicht allerdings nicht aus, um ein Produkt als zulässiges Lebensmittel deklarieren zu können. Vielmehrmussessichumein imLebensmittelrechtumschriebenes Lebensmittel handeln. ${ }^{123}$ Die zulässigen Arten von Lebensmitteln und die entsprechenden Vorgaben werden in gesonderten Verordnungen geregelt. ${ }^{124}$ Für nicht umschriebene Lebensmittel muss eine Bewilligung des Bundesamtes für Gesundheit eingeholt werden. ${ }^{125}$ Als mögliche Gruppe für die Frauenmilch kommen die Speziallebensmittel oder Lebensmittel tierischer Herkunft in Betracht. ${ }^{126}$ Die Speziallebensmittelgesetzgebung erfasst indes nur Säuglingsanfangsnahrung, die auf künstlicher Basis hergestellt wurde, weshalb die natürliche Frauenmilch nicht in deren Geltungsbereich fällt. ${ }^{127}$ Milch wird grundsätzlich von der Verordnung über Lebensmittel tierischer Herkunft erfasst, Frauenmilch jedoch nicht. In der biologischen Systematik gehört der Mensch zwar zu der Gruppe der Säugetiere, rechtlich ist der Mensch aber kein Tier. ${ }^{128}$ Der Mensch kann damit nicht zu den zugelassenen (Tier-)Arten für die Lebensmittelgewinnung gezählt werden. ${ }^{129}$

119 Poledna/Berger, S. 36; Frick, S. 234 ff.

${ }^{120}$ Art. 2 Bundesgesetz über Lebensmittel und Gebrauchsgegenstände (LMG) vom 9. Oktober 1992, SR 817.0. Ausführlich zum Inverkehrbringen von Lebensmitteln POLEDNA, S. $41 \mathrm{ff}$

${ }^{121}$ Botschaft zu einem Bundesgesetz über Lebensmittel + Gebrauchs gegenstände (BBI 1989 I, S. 893 ff.), S. 893 ff; POLEDNA, S. 43.

122 Art. 3 LMG, vgl. auch BBI 1989 I, S. 913; siehe auch Urteil 2A.565/2000.

${ }^{123}$ Art. 4 Lebensmittel- und Gebrauchsgegenständeverordnung (LGV) vom 23. November 2005, SR 817.02

124 Z.B. die Verordnung des EDI über Speisepilze und Hefe vom 23 November 2005, SR 817.022.106, oder die Verordnung des EDI über alkoholfreie Getränke vom 23. November 2005, SR 817.022.11.

125 Art. 8 LMG i.V.m. Art. 5 Abs. 1 LGV.

126 Verordnung des EDI vom 23. November 2005 über Speziallebensmittel, SR 817.022.104; Verordnung des EDI vom 23. November 2005 über Lebensmittel tierischer Herkunft, SR 817.022.108.

127 Art. 2 Abs. 2 lit. $m$ und Art. 17 ff. Verordnung über Speziallebensmittel.

${ }_{128} \mathrm{Vgl}$. auch Art. 11 und Art. 641a ZGB.

${ }^{129} \mathrm{Vgl}$. Art. 67 und Art. 2 der Verordnung über Lebensmittel tierischer Herkunft.
[Rz 50] Ein wichtiger Schutzmechanismus der Lebensmittelgesetzgebung ist die Qualitätskontrolle. Sie wird in der Schweiz durch Selbstkontrolle und durch kantonale Kontrollen gewährleistet. ${ }^{130}$ Bei der Selbstkontrolle wird auf den Standard der "guten Herstellungspraxis» verwiesen. Für Milchbanken ist in der Schweiz keine "gute Herstellungspraxis» in Form von Richtlinien definiert. In Deutschland existieren seit 1998 Leitlinien für die Einrichtung und die Arbeitsweise von Frauenmilchbanken. Die entsprechenden Verfahren und Vorschriften zur Qualitätssicherung werden darin ausführlich beschrieben. ${ }^{131}$ Die meisten schweizerischen Milchbanken orientieren sich an diesen Leitlinien. Sie können deshalb «gewohnheitsrechtlich» als schweizerische "gute Herstellungspraxis» angesehen werden.

[Rz 51] Die Frauenmilch kann also gegenwärtig weder als Arzneimittel noch als Lebensmittel bezeichnet werden. Diese Rechtsunsicherheit liesse sich mit einem einmaligen Gesuch um Aufnahmein die Reihe derumschriebenen Lebensmittel beheben, was jedoch für die Gesuchstellenden mit einigem Aufwand verbunden ist. Im Moment ist die Erwirkung einer Bewilligung noch nicht akut, doch wird diese lebensmittelrechtliche Frage spätestens dann zu klären sein, wenn unter den Spitälern ein Austausch an Frauenmilch stattfinden wird. Es entspräche zudem der ratio legis, dem Schutz der Konsumenten, die Milch als Lebensmittel zu deklarieren.

[Rz 52] Die Milchbanken dürften die Anforderungen der Lebensmittelgesetzgebung bezüglich des Umgangs mit Lebensmitteln allerdings problemlos erfüllen; dies nicht zuletzt deshalb, weil die Milchbanken fest in die Spitalorganisation integriert sind und somit deren hohen hygienischen Standard und die Standardisierung der Arbeitsabläufe inkorporiert haben. Die lebensmittelrechtlichen Bewilligungs- und Meldepflichten wären deshalb blosse Formsache.

\section{Abschliessende Gedanken}

[Rz 53] Es ist an und für sich ein gutes Zeichen, dass sich bislang trotz des Betriebs einiger Frauenmilchbanken in der Schweiz noch keine rechtlichen Fragen gestellt haben. Gleichwohl erscheint es angemessen, diesen Institutionen etwas mehr juristische Beachtung zu schenken: Einerseits weil sie wichtige Leistungen im Interesse der Frühgeborenen und allenfalls anderen Empfängerinnen und Empfängern erbringen, anderseits weil sich - im Hinblick auf den Schutz der Frühgeborenen und einen allfälligen Austausch von Frauenmilch zwischen den Institutionen - gewisse Sicherheits- und Schutzstandards aufdrängen.

[Rz 54] Im Hinblickauf die Kostenreduktion im Gesundheitswesen und die damit verbundene Verschiebung «vom Heilen zur Prävention» dürfte die Institution der Milchbank an Bedeutung gewinnen. Im Hinblick auf das Kindeswohl wäre es wünschenswert, die für die Säuglinge optimale Ernährung einem

\footnotetext{
${ }^{130}$ Art. 23 lit. f und 32 Abs. 3 LMG.

131 SPRINGER, passim.
} 
möglichst grossen Kreis zugänglich zu machen. Dies bedingt eine Förderung und Anerkennung der Frauenmilchspende. Rechtliche Hürden, die eine Spende unattraktiv machen, sollten nach Möglichkeit beseitigt werden.

\section{Literaturverzeichnis}

Die folgenden Werke werden, sofern nicht anders angegeben, mit dem/den Namen des Autors/der Autoren sowie der Seitenzahl oder Randnummer (N.) zitiert.

Ach Johann S./Anderheiden Michael/Quante Michael, Ethik der Organtransplantation, Erlangen 2000.

Aubert Jean-Francois/Mahon Pascal (Hrsg.), Petit commentaire de la Constitution fédérale de la Confédération Suisse du 18 avril 1999, Zurich/Bâle/Genève 2003.

Ball Thomas M./WRight Anne L., Health care costs of formula-feeding in the first year of life, in: Pediatrics, Vol. 103, No. 4, April 1999, 870 ff.

Both DeNISE, Frauenmilchbanken - jeder Tropfen zählt, in: Laktation und Stillen 1, 2006, S. 4 ff.

Breining-Kaufmann Christine, Hunger als Rechtsproblem - völkerrechtliche Aspekte eines Rechts auf Nahrung, Zürich 1991.

Bundesamt Für Statistik BFS (Hrsg.), Neugeborene in Schweizer Spitälern 2004, Spitalversorgung von termin- und frühgeborenen Säuglingen, Bern 2007 (zit. BFS, S. ...).

Frick Markus R., Werbung für Lebensmittel, in: Lebensmittelrecht, Poledna Tomas/Arter Oliver/Gattiker Monika (Hrsg.), Bern 2006, S. 245.

GÄchter ThOMAS, Soziale Grundrechte, Das nackte Überleben oder mehr? in: lus.full, Nr. 3+4/2007, S. 138 ff. (zit. GÄCHTER, Soziale Grundrechte).

GÄ́CHTER THOMAS, Grenzen der Solidarität? in: Individuum und Verband, Festgabezum Schweizerischen Juristentag 2006,Zürich/ Basel /Genf 2006, S. 473 ff. (zit. GÄCHTER, Grenzen der Solidarität).

Guillod Olivier/Sprumont Dominique, Le droit à la santé: un droit en émergence, in: De la constitution, Etudes en l'honneur de JeanFrançois Aubert, Basel 1996, S. 337 ff.

HaAb Robert, in: BürgI F./Homberger A./Schönenberg W. et al. (Hrsg.), Kommentar zum Schweizerischen Zivilgesetzbuch, Das Sachenrecht, 2. Aufl., Zürich 1977. S. $11 \mathrm{ff}$.

Häfelin Ulrich/Haller Walter, Schweizerisches Bundesstaatsrecht, 6. Aufl., Zürich/Basel/Genf 2005.

Hodgkin Rachel/Newell Peter, Implementation Handbook for the Convention on the Rights of the Child, UNICEF (Hrsg.), New York/ Geneva 2002.

KÄLIN OuIveR, Der Sachbegriff im schweizerischen ZGB, Zürich/ Basel/Genf 2002

KÄLIN WALTER/KÜNZLI JÖRG, Universeller Menschenrechtsschutz, Basel 2005.
Kayser Martin/Richter Dagmar, Die neue schweizerische Bundesverfassung, in: Zeitschrift für ausländisches öffentliches Recht und Völkerrecht (ZaöRV) 1999, S. 985 ff.

KrameR M. ET AL, Promotion of Breastfeeding Intervention Trial: A randomized trial in the Republic of Belarus, in: Journal of the American Medical Association, 2001, 285 (4), S. 413 ff.

Künzlı JÖRG/KäLIN WaLter, Die Bedeutung des UNO-Paktes über wirtschaftliche, soziale und kulturelle Rechte für das schweizerische Recht, in: Kälin Walter/MalinvernI Giorgio/NowaK Manfred (Hrsg.), Die Schweiz und die UNO-Menschenrechtspakte, Basel 1997, S. $105 \mathrm{ff}$.

LANDOLt HaRDY, Pflegerecht, Band I: Grundlagen des Pflegerechts, eine Darstellung der begrifflichen, statistischen und volkswirtschaftlichen Grundlagen und des internationalen Pflegerecht, Bern 2001

LUCAS A./COLETJ.,Breast milk and neonatal necrotising enterocolitis, in: The Lancet: 1990, Vol. 336, S. 1519 ff.

LuCAS A/Morley R/CoLeTJ/GoreSM, A randomised multicentre study of human milk versus formula and later development in preterm infants, in: Archives of Disease in Childhood Fetal and Neonatal Edition, March 1994, 70 (2).

Maurer Alfred, Bundessozialversicherungsrecht, 2. Auflage, Basel/ Frankfurt am Main 1994.

Merhav HJ/Wright HI/Miles LA/VAn Thiel. DH, Treatment of IgA deficiency in liver transplant recipients with human breast milk, in: Transplantation International, 1995, 8 (4).

NowAK MANFRED, Inhalt, Bedeutung und Durchsetzungsmechanismen der beiden UNO-Menschenrechtspakte, in: KäLIN Walter/Malinverni Giorgio/Nowak Manfred (Hrsg.), Die Schweiz und die UNO-Menschenrechtspakte, Basel 1997, S. 3 ff.

Pelet Odile, Organes, tissues, cellules: loin du corps, loin de la personne?, Bern 2002.

Pfiffner Rauber BrigitTe, Das Recht auf Krankheitsbehandlung und Pflege. Zum Behandlungsanspruch von Krankenversicherten im RahmenderWirtschaftlichkeitunterbesondererBerücksichtigung der Langzeitpflege, Diss. (Zürich), Zürich/Basel/Genf 2003.

POLEDNA TOMAS, Inverkehrbringung von Lebensmitteln und Lebensmittelkontrolle, in: Lebensmittelrecht, Poledna Tomas/Arter Oliver/ GATTIKER MONIKA (Hrsg.), Bern 2006, S. 41 ff.

Poledna Tomas/Berger BrigitTe, Öffentliches Gesundheitsrecht, Bern 2002.

Rey Heinz, Die Grundlagen des Sachenrechts und das Eigentum, Grundriss des schweizerischen Sachenrechts, Band I, 2. Aufl., Bern 2000.

RHINOW ReNÉ, Grundzüge des Schweizerischen Verfassungsrechts, Basel 2003.

Schmid JÖRg/HürLimann-Kaup Bettina, Sachenrecht, Zürich/Basel/ Genf 2003. 
SCHREBBER KLAuS, Sachenrecht, 4. Aufl., Stuttgart/München/ Hannover/Berlin/Weimar/Dresden 2003.

Singhal Atul/Cole Tim J/Luca Alan, Early nutrition in preterm infants and later blood pressure: two cohorts after randomised trials, in: The Lancet, 2001, Vol. 357, S. $413 \mathrm{ff}$.

SPRINGER SKADI, Leitlinie für die Einrichtung und zur Arbeitsweise von Frauenmilchbanken, Leipzig 1998.

Steiner Christoph, Das Recht auf soziale Gesundheitsversorgung, Frankfurt am Main 2004.

Tully MARY Rose, Stories of Success: The Use of Donor Milk is increasing in North America, in: Journal of Human Lactation, Volume 20, N 1, 2004, S. $75 \mathrm{ff}$.

Tuor Peter/Schnyder Bernhard/Schmid Jörg/Rumo-Jungo Alexandra, Das Schweizerische Zivilgesetzbuch, 12. Auflage, Zürich/Basel/Genf 2002.

WaLdMANn BerNHARD, Das Recht auf Nothilfe zwischen Solidarität und Eigenverantwortung, in: Schweizerisches Zentralblatt für Staats- und Verwaltungsrecht 7/2006, S. $341 \mathrm{ff}$.

WHO/UnICEF, Global Strategy for Infant and Young Child Feeding, Genf 2003, www.who.int/child-adolescent-health/New Publications/NUTRITION/gs_iycf.pdf (besucht am 15.5.06) (zit. WHO, Global Strategy, S. ...).

WHo, The Optimal Duration of Exclusive Breastfeeding, Genf 2002, www.who.int/nutrition/publications/optimal_duration_of exc_bfeeding_review_eng.pdf (besucht am 17.07.07) (zit. WHO, Breastfeeding, S. ...).

Wiegand Wolfgang, Kommentar zu Art. 641 ff., in: Honsell Heinrich/ Vogt Nedim Peter/Geiser Thomas (Hrsg.), Zivilgesetzbuch II, Art. 457-977 ZGB, Art. 1-61 SchlT ZGB, Basler Kommentar, 2. Auff. Basel/Genf/München 2003, S. 778 ff., N. 1 ff.

Wight NANCY E, Donor Human Milk for Preterm Infants, in: Journal of Perinatology, June 2001, Volume 21, N. 4, S. 249 ff.

Work Group on Breastfeeding, American Academy of Pediatrics, Breastfeeding and the use of Human Milk, in: Pediatrics, Vol 100 No. 6, December 1997, S. 1035 ff.

Zillgen Barbara, Die strafrechtlichen Grenzen der Lebendorganspende, Betrachtungen de lege lata und de lege ferenda, Frankfurt am Main 2004
MLaw (Luzern) Caroline Brugger SChmidt ist wissenschaftliche Assistentin am Lehrstuhl für Staats-, Verwaltungs- und Sozialversicherungsrecht der Universität Zürich, Prof. Dr. iur. THOMAS GÄCHTER ist Inhaber dieses Lehrstuhls und ständiger Gastprofessor für Sozialversicherungs- und Gesundheitsrecht an der Universität Luzern. Die Abhandlung basiert auf einem Referat an der Herbsttagung 2006 der Schweizerischen Gesellschaft für Neonatologie in Basel. Wir danken Frau lic. iur. EvA SIKI für die sorgfältige Durchsicht des Manuskripts. 